\title{
What do pediatric surgeons think about sexual issues in dealing with patients with anorectal malformations? The ARM-Net consortium members' opinion
}

\author{
Eva Elisa Amerstorfer ${ }^{1}$. Caterina Grano $^{2}$. Chris Verhaak ${ }^{3}$. Araceli García-Vasquez ${ }^{4}$ Marc Miserez $^{5}$. \\ Annemone Radleff-Schlimme ${ }^{6} \cdot$ Nicole Schwarzer $^{6} \cdot$ Michel Haanen $^{7} \cdot$ Ivo de Blaauw $^{8} \cdot$ Ekkehart Jenetzky $^{9,10}$.

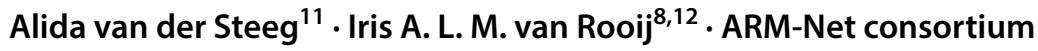

Accepted: 20 June 2019 / Published online: 5 July 2019

(c) The Author(s) 2019

\begin{abstract}
Purpose Since pediatric surgeons aim to follow their patients with anorectal malformations (ARM) into adulthood the aim of this study was to investigate how pediatric surgeons deal with sexual issues related to ARM.

Methods In 2018, a questionnaire was developed by the working group "Follow-up and sexuality" of the ARM-Net consortium and sent to all consortium-linked pediatric surgeons from 31 European pediatric surgical centers. Obtained data were statistically analyzed.

Results Twenty-eight of 37 pediatric surgeons (18 males/10 females) answered the questionnaire. The majority of pediatric surgeons (82\%) think they should talk about sexual issues with their patient. More than 50\% of pediatric surgeons do not feel at all or only moderately confident discussing the topic of sexuality. Most pediatric surgeons require more support (96\%) and wish to be trained in sexuality and sexual issues (78\%) to feel confident towards their ARM-patients/parents. For optimal care, sexual issues with ARM-patients should be managed by a multidisciplinary team.

Conclusions Pediatric surgeons feel that sexuality is an important issue for their ARM-patients, which they are primarily responsible of but should be managed in concert with a multidisciplinary team. A training in sexuality is wished to feel more confident about this specific issue.
\end{abstract}

Keywords Anorectal malformation $\cdot$ Sexuality $\cdot$ Sexual functioning $\cdot$ Adolescence $\cdot$ Training $\cdot$ ARM-Net

\section{Introduction}

Sexual development already starts in childhood, when toddlers start to explore their body and interact with other children by inducing games such as "doctor" and "patient", building up their sexual identity [1]. An impaired body

Eva Elisa Amerstorfer and Caterina Grano share first-authorship.

Eva Elisa Amerstorfer, Caterina Grano and Iris A. L. M. van Rooij are contributed equally to the work.

The members belonging to the ARM-Net consortium not mentioned as contributors are written down below the "Acknowledgements" section.

Eva Elisa Amerstorfer

ee.amerstorfer@gmail.com

Extended author information available on the last page of the article image, as well as impaired self-esteem, problems with incontinence, dependence on parental help and other psychosocial factors may interfere with normal sexual development during childhood and adolescence [2-5]. These factors might be the reason that a significant number of adult patients with an anorectal malformation (ARM) is dissatisfied with their partnership and sexuality $[5,6]$ or report some forms of sexual dysfunction [7, 8]. Patients with an ARM undergo reconstructive surgeries during infancy or childhood and often suffer from remaining perineal or abdominal scars as well as sustained fecal and urinary incontinence or constipation. Depending on the degree of symptoms, quality of life (QoL), social life, psychological morbidity, as well as sexual health are affected in this patient cohort during childhood, adolescence and adulthood [4, 5, 9-13]. Though, not only might sexuality itself be a major issue to patients with ARM, it has been further stated that in adult male ARM patients difficulties in close and sexual relationships are predictive 
for depressive feelings [14]. Thus, as it has been stressed by others, it is paramount that beside anatomic reconstruction, sexual function is preserved and that ARM patients can achieve independence from their parents, become self-efficient and are able to establish contact with peers to develop satisfying and prosperous intimate and sexual relationships $[4,5]$.

The precise anatomic reconstruction and preservation of organic function are the primary targets of pediatric surgeons in treating patients with ARM. Nowadays, many pediatric surgeons follow their patients into adulthood and experience problems in the transfer from adolescent to adult care regarding sexual health. In that context, an interview by sexologists with adolescents with ARM revealed that the patients wished to be contacted and informed about possible sexual problems related to their disease by their pediatric surgeon during follow-ups [13]. However, as demonstrated by a recent study, only a few patients are educated about sexual issues related to ARM during medical care, although more than half of the investigated patients would have appreciated that [7]. Talking freely about sexual issues, and evaluating sexual function, however, represents a peculiar situation for patients and their physicians. Reasons for infrequent communication may include marginal training of surgeons on intimate relationships and sexual issues and hesitancy of the patients due, for example, to potential embarrassment [15].

The goal of the present study was therefore to investigate how pediatric surgeons deal with sexual issues related to ARM. More specifically, we were interested in understanding if pediatric surgeons feel that they should talk of sexual issues with their ARM patients, if they feel confident in doing that, if their confidence differs depending on the patient's gender, at what age sexual issues should be discussed with the patient, if they wished to be more trained in sexuality and sexual issues, and which professionals they think should be included in managing patients with ARM during their sexual development.

\section{Methods}

In 2010, several European pediatric surgical centers involved in the care for patients with ARM founded the ARM-Net consortium, involving pediatric surgeons, epidemiologists, psychologists, geneticists, and representatives of patient organizations [16]. The aim of this consortium is to exchange data and knowledge to improve clinical care and to perform research into genetic, epidemiological and clinical subjects as well as registering and monitoring frequencies of types of ARM, additional malformations, surgical procedures, complications, and outcomes. In addition, its focus is to harmonize diagnostic classification, possible interventions, and follow-up to improve QoL of ARM patients.

\section{Questionnaire}

A questionnaire evaluating sexual issues was developed by the working group "Follow-up and sexuality" of the ARMNet consortium based on analyses of previous literature conducted in the field of sexuality in ARM patients, and on relevant information given by pediatric surgeons and by patients and parents associations represented in the ARMnet consortium. The final questionnaire was composed of 21 items. Examples of items of the questionnaire were: "Do you address questions about sexual issues to your patients during follow-up?" answered on a dichotomous Yes/No scale or "Do you feel prepared to talk about sexuality with your ARM patients?" answered on a five-point response scale ranging from one (not at all) to five (extremely).

\section{Procedures}

All the pediatric surgeons $(N=37)$ belonging to the ARMNet consortium received an e-mail containing a weblink with the final online version of the questionnaire implemented through the Survey monkey provider. Once the link was opened, a brief presentation of the study was given and participants were asked to give their informed consent, before starting filling in the questionnaire. The questionnaire was sent by e-mail a first time in March 2018 with a first recall in April and a last recall in September of the same year.

\section{Statistical analyses}

All the questionnaires were exported from the Survey monkey provider as an Excel file. The database was cleaned (eliminating duplicates and empty cases) and transformed in a SPSS file. The program IBM Statistics SPSS 25 was used to analyze the data. Descriptive statistics [mean values, standard deviations (SD), or if more appropriate median and range, and percentages] were calculated. For some questions the percentages are provided for male and female pediatric surgeons separately to consider possible different perspectives.

\section{Results}

Of the 37 pediatric surgeons contacted, 28 answered the questionnaire for a response rate of $76 \%$. A total of 24 pediatric units participated in the study, from four units two pediatric surgeons participated. The respondents came from 11 different European countries, namely Austria, Belgium, Germany, Israel, Italy, The Netherlands, Spain, Sweden, 
Switzerland, Ukraine, and United Kingdom (Table 1). More males than females participated and the mean age at filling in the questionnaire was 47 years. The number of patients the pediatric units reported to see each year at their clinic ranges from 15 to 250 of which 3-30 are new patients. The number of adolescent or adult patients is much lower and ranges from 2 to 60 and approximately $25 \%$ of them are new each year and the rest is followed-up.

Results from the questions asked to the pediatric surgeons about their current practice on the issue of sexuality are reported in Table 2. Most ARM patients are followed-up into their adolescence. In 54\% pediatric surgeons screened for sexual function from infant age onwards, but only three of them used standardized questionnaires for this. Of these three, one used the Mathtech questionnaire [17] and two used a questionnaire developed by themselves, one of them derived from a general national questionnaire for adolescents that was adapted for ARM patients. Half of the participating pediatric surgeons asked questions about sexual experiences to adolescent or adult ARM patients and 39\% of the patients talked about sexuality in general, but none of them often. There is no difference found between male or female pediatric surgeons on these issues. In most pediatric units (82\%) a psychologist was part of the team, working with ARM patients in the hospital, and in 50\% these psychologists were dedicated to ARM patients. A sexologist was only present in the team in one-fifth of all pediatric units and was often not dedicated to ARM patients.
The majority of pediatric surgeons specified that the issue of sexuality is important for ARM patients and that they should talk about this issue with their patients (Table 3). A smaller group of surgeons, but still 64\% thinks that they should be the first responsible ones to address sexual function with patients, with a difference in opinion between female (80\%) and male pediatric surgeons (56\%). The age at which to start to discuss questions about sexuality was suggested to be between 11 and 17 years, with a mean of 14.6 years (SD:1.6). The idea of $50 \%$ of the pediatric surgeons is that adolescent or adult patients would like to talk about sexuality with them often or very often if they were given the opportunity. The same idea of the pediatric surgeon is true for the parents talking about the sexuality of their child.

When we asked about the feeling of confidence and being prepared to talk about sexuality, the opinions of the respondents differed quite a lot, and more than 50\% do not feel at all or only moderately confident discussing the topic of sexuality. Women more often (70\%) scored a lowto-moderate feeling of confidence compared to men (45\%). Five pediatric surgeons ( 4 males and 1 female) mentioned that their confidence would differ depending on the ARM patient's gender.

Almost all pediatric surgeons would need support providing consultation on sexual issues to their ARM patients and $78 \%$ wish to be trained for that. The majority is of the opinion that other professionals should also face the
Table 1 Characteristics of the participating pediatric surgeons and pediatric units

\begin{tabular}{ll}
\hline Gender pediatric surgeons, $N(\%)$ & $18(64.3)$ \\
Male & $10(35.7)$ \\
Female & $46.9(7.7)$ \\
Age pediatric surgeon, mean (SD) & \\
Number of patients seen per year in a pediatric unit, median (range) & $55(15-250)$ \\
Total number of ARM patients & $7(2-60)$ \\
Number of adolescent/adult ARM patients & $8(3-30)$ \\
Number of new ARM patients & $2(0-15)$ \\
Number of new adolescent/adult ARM patients & \\
Number of pediatric units per country, N/number of patients seen each year, mean (SD) & $2 / 64(51)$ \\
Austria & $1 / 15$ \\
Belgium & $4 / 29(15)$ \\
Germany & $1 /$ missing \\
Israel & $5 / 136(87)$ \\
Italy & $3 / 152(84)$ \\
The Netherlands & $3 / 75(35)$ \\
Spain & $2 / 80(87)$ \\
Sweden & $1 / 25$ \\
Switzerland & $1 / 20$ \\
Ukraine & $1 / 20$ \\
United Kingdom & \\
\hline
\end{tabular}

$A R M$ anorecal malformation, $S D$ standard deviation 
Table 2 Current practice on the issue of sexuality in the care of ARM patients by pediatric surgeons
$N(\%)^{\mathrm{a}}$

Do you follow your ARM patients into their adolescence?

Yes, all of them

$15(57.7)$

Yes, most of them

$9(34.6)$

Yes, but only a small part of them

$2(7.7)$

No

$0(0.0)$

Do you screen for sexual function ${ }^{\mathrm{b}}$ from infant age (1 year of age) onwards?

Yes

$15(53.6)$

No

$13(46.4)$

Do you use any standardized questionnaires for asking about sexuality?

Yes

No

$25(89.3)$

Do you address questions concerning sexual experiences to your adolescent/adult ARM patients during follow-up visits?

Never

Seldom

Sometimes

Often

$5(17.9)$

Very often

$2(7.1)$

Do your ARM patients ask you questions about sexuality?

Never

Seldom

Sometimes

$11(39.3)$

Often

$0(0.0)$

Very often

Do you have a psychologist in your team at the hospital?

Yes

No

If yes,

Is he/she dedicated to the ARM patients?

Yes

No

Is he/she a pediatric psychologist?

Yes

No

I don't know

Do you have a sexologist in your team at the hospital?

Yes

No

If yes,

Is he/she dedicated to the ARM patients?

Yes

No

Does he/she work also with children/adolescents?

Yes

No

ARM anorectal malformation

${ }^{\text {a }}$ Percentages are calculated from those who responded to the question, so ignoring missing answers

${ }^{b}$ For example erection in neonatal age, secondary characteristics during puberty 
Table 3 Opinions about practice on the issue sexuality in ARM patients by pediatric surgeons

$N(\%)^{\mathrm{a}}$

Do you think sexuality is an important issue for ARM patients?

$\begin{array}{lc}1 \text {-not at all important } & 0(0.0) \\ 2 & 1(3.6) \\ 3 & 1(3.6) \\ 4 & 8(28.6) \\ 5-\text { extremely important } & 18(64.3) \\ \text { Do you think the pediatric surgeon should talk about sexual issues with his/her patients? } & 23(82.1) \\ \text { Yes } & 5(17.9)\end{array}$

Do you think, if they were given the possibility, your adolescent or adult patients/their parents would like to talk more about Patients/parents sexuality with you?

Never

$0(0.0) / 0(0.0)$

Seldom

$4(14.3) / 5(17.9)$

Sometimes

$10(35.7) / 7(25.0)$

Often

$12(42.9) / 15(53.6)$

Very often

2 (7.1)/1 (3.6)

Do you feel prepared to talk about sexuality with your ARM patients/their parents?

$2(7.1) / 1(3.6)$

Not at all

$2(7.1) / 2(7.1)$

A little

7 (25.0)/6 (21.4)

Moderately

$6(21.4) / 7$ (25.0)

Quite well

$13(46.4) / 12(42.9)$

Extremely

0 (0.0)/1 (3.6)

How much confidence do you feel in talking about sexuality with your ARM patients/their parents?

Patients/parents

1 -not at all confident

$1(4.0) / 1(3.6)$

2

$5(20.0) / 4(14.3)$

3

$8(32.0) / 9(32.1)$

4

$9(36.0) / 13(46.4)$

5-very confident

$2(8.0) / 1(3.6)$

Do you have the feeling that, for your consultation with ARM patients and their parents, you need more support regarding sexual issues?

Yes

$26(96.3)$

No

$1(3.7)$

Would you, as a pediatric surgeon, wish to be trained and advised regarding sexuality and sexual issues of ARM patients?

Yes

$21(77.8)$

No

$6(21.4)$

Do you think other professionals other than the pediatric surgeon should face sexual issues with ARM patients?

Yes

$26(92.9)$

No

$2(7.1)$

Who would be suitable to discuss sexual issues with ARM patients?

Pediatric surgeons

$18(64.3)$

Adult surgeon, expert of ARM

$9(32.1)$

Gynecologist/andrologist

27 (96.4)

Sexologist/psychologist

25 (89.3)

Nurse

13 (46.4)

Other $^{\mathrm{b}}$

$7(25.0)$

$A R M$ anorectal malformation

${ }^{\text {a }}$ Percentages are calculated from those who responded to the question, so ignoring missing answers

${ }^{b}$ Others mentioned were: pediatric urologist, adult urologist, physiotherapist, pediatrician, multidisciplinary team with the surgeon involved 
subject of sexuality of the ARM patients, especially the gynecologists or andrologists, and the psychologists or sexologists.

\section{Discussion}

In 2010, the WHO has defined sexual health as a state of physical, emotional, mental and social well-being, which requires a positive and respectful approach to sexuality as well as sexual relationships and is given the opportunity to enjoy pleasurable and safe sexual experiences that are free of discrimination, coercion, and violence [18]. For attainment and maintaining of sexual health, sexual rights of each individual have to be respected, protected, and fulfilled [18]. Sexual health influences the overall health of an individual and has thus a significant impact on one's QoL. With the awareness, that evaluation, attainment and maintenance of sexual health are especially lacking in patients with the history of a congenital ARM, the ARM-Net consortium aimed to identify current practice, as well as personal opinions of pediatric surgeons from different European renown pediatric surgical centers towards this specific issue. As demonstrated by a recent review, pediatric surgeons have by now recognized their responsibility to follow their patients with ARM into adulthood [19]. This approach coincides with the fact that more than $90 \%$ of the pediatric surgeons who participated in this study follow their ARM patients. However, only half of them screen them for sexual function from neonatal age onwards. This finding reflects that many surgeons are nowadays concerned about urological and bowel function in the postoperative management of ARM, but are less aware of inquiring sexual function as a routine parameter during follow-ups. It has been stated in literature that associated urological and gynecological anomalies may even be missed in the newborn period or at the definitive surgery of ARM, unless the treating surgeon is aware of this association [20, 21].

The majority of female patients with the most complex form of ARM, the cloacal anomaly, was reported to have healthy sexual relationships and may become pregnant $[22$, 23]. However, recent studies demonstrated that females with ARM experience more frequent sexual dysfunctioning and sexual distress compared to a reference population [7, 8]. Indeed, gynecologic problems during puberty are not uncommon. Pubescent girls with the history of ARM may experience amenorrhea due to an absence or underdevelopment of Müllerian structures or suffer from obstruction to menstrual flow due to a non-patent outflow tract [21]. Further gynecologic problems such as missed vaginal anomalies, hydrosalpinges, adnexal cysts, endometriosis, and chronic pelvic pain associated with endometriosis or pubertal stimulation of chronically adhesed reproductive anatomy may significantly harm female ARM patients during adolescence [21]. Thus, it has been recommended to perform a complete physical examination after puberty that includes an assessment of secondary sexual development and determination of the adequacy of the reproductive anatomy to ascertain sexual intimacy and avoid adverse sequelae [21]. Eventually, parents already would like to learn about the reproductive potential of their child when they are confronted with the diagnosis of ARM, which stresses the need for appropriate counseling of patients and parents to allow optimal sexual development as well as preservation of future fertility.

It is known from literature that males with ARM appear to have a preserved erectile and orgasmic function after being treated by posterior sagittal anorectoplasty, but may suffer from absent ejaculations or azoospermia $[8,24]$. In contrast, in male patients treated by abdominoperineal endorectal pull-through (Rehbein's procedure) or abdominosacroperineal endorectal pull-through for high or intermediate type ARM, the incidence of sexual problems such as erectile and ejaculatory dysfunction was described with a percentage of $40 \%$ [25]. These findings also announce the importance to follow patients with ARM into their adulthood, and to evaluate sexual development to provide each individual with ARM, counselling and support when sexual distress occurs.

In this context, this study demonstrates that most pediatric surgeons consider sexuality as an important issue to ARM patients and also do think that it is their responsibility to address questions about sexual issues to their ARM patients. A standardized questionnaire evaluating sexual health in adolescents with ARM is, however, lacking and only a small cohort of pediatric surgeons used specific questionnaires evaluating sexual aspects. Nevertheless, half of the participating pediatric surgeons reported that they ask questions about sexual experiences to their adolescent ARM patients during follow-up visits. On the other hand, many physicians lack the comfort, expertise and willingness to inquire intimate sexual aspects from their patients [15]. This is in alignment with the findings of this study that more than $50 \%$ of the pediatric surgeons do not feel at all or only moderately confident discussing the topic of sexuality with their patients and that almost all pediatric surgeons feel that they need more support for consulting their patients and their parents towards sexual issues and actually, wish to be trained in sexuality and sexual issues related to ARM. In fact, it has been stated that physicians feel insecure and reluctant about this vulnerable topic and thus, do not address sexual health proactively as they fear to open a "can of worms" $[26,27]$. This may be due to the lack of medical training in human sexuality, which may cause discomfort in talking about sexual issues in doctors [15].

On the other way, it is still infrequent that patients raise a question about sexuality to their surgeon. This finding is 
most likely linked to the reported hesitancy of patients with chronic illness, which may cause embarrassment and create a barrier to patient-doctor discussion about sex [15]. Furthermore, our findings document that women seem to feel less confident than men in addressing questions about sexuality and that, on the opposite, more men than women believe that their confidence also differs depending on the ARM patient's gender. Indeed, talking about sexual issues seems to be more comfortable in a same-gender consultation which is preferred by health professionals as well as patients, as demonstrated in a survey from the United Kingdom [26]. Reasons for this preference were stated as concerns that patients of the opposite gender may sexualize the consultation [26]. Apart from that, a tendency that patients self-select health professionals along gender lines was also noted [26]. Thus, health providers should be aware of these problems and find ways to deal with them during the consultation.

In agreement with the wish for a specialized training in sexuality and sexual issues of the majority of pediatric surgeons from this study, it has been recently exclaimed that there is an urgent need for an up-to-date sexual health education not only for present-day physicians, but also for medical students and patients to improve sexual health care $[27,28]$. In this context, effective sexual training programs that are designed to build up professional capacity should integrate biological, psychological as well as social aspects of sexuality [28].

In an optimal setting to assess sexual health and manage sexual problems of patients with ARM, pediatric surgeons collaborate with a team of other professions such as gynecologists, andrologists, adult colorectal surgeons, pediatricians, pediatric/adult urologists, psychologists, sexologists, physiotherapists and nurses, which was claimed by the majority of pediatric surgeons. Especially, dedicated psychologists and sexologists with knowledge of ARM may provide substantial support when patients experience sexual dysfunction and dissatisfaction and have problems to start and maintain sexual relationships. Even though, a psychologist, who is in $70 \%$ a pediatric psychologist and in 50\% dedicated to patients with ARM, is part of the team in most pediatric surgical centers, the possibility to include a sexologist in counselling patients with sexual problems is still uncommon.

One has to keep in mind, that sexual function may be impaired long before a patient with chronic illness reaches puberty, which has an impact on future sexual relationships as well as sexual satisfaction which significantly influences one's QoL [15]. The ideal age to investigate sexual health during follow-up has been suggested at a mean age of 14.6 years in this study. Breech has recommended to evaluate sexual function in girls after puberty and before the onset of sexual activity, which allows the treating physician to recognize and treat associated urological and gynecological anomalies and their long-term sequelae to enable sexual intimacy [21]. In terms of perceived self-competence during adolescence, having romantic relationships and close friendships in childhood was related to sexual desire and erectile function in male patients with ARM during adulthood, whereas, scholastic competence, physical appearance and global self-worth in childhood negatively predicted sexual distress in female ARM patients during adulthood [8]. These concerns announce the need to address not only physical aspects of sexuality, but also psychosocial and emotional aspects of sexuality during follow-up visits at an adolescent age, which may be best managed in the setting of a transitional outpatient clinic that is involving a multidisciplinary team [8]. In line with the UNESCO guidelines from 2018 [29], educating patients and parents about sexual intimacy should already be encouraged from the age of 5-8 years in an age-appropriate language and way. From a personal view of a patient with ARM from the ARM-Net consortium, this enables the patient to learn about his/her body and accept traumatized parts of the body as their own. This early confrontation with sexual intimacy may eventually help to build up the patient's body image, but may also create a basis to be able to talk about sexual concerns in a trustful way later on.

In conclusion, this study revealed that pediatric surgeons consider sexuality an important issue to ARM patients and their parents and think that it is their primary responsibility to address questions about sexual issues to their patients. It is of great importance that pediatric surgeons dedicated to ARM provide an adequate continuous support and counselling in concert with a multidisciplinary team, allowing a patient with a congenital ARM to experience "normal" sexual development, achieve sexual health and enjoy sexual relationships later on. To achieve this goal and provide better care, pediatric surgeons who treat patients with ARM shall receive medical training regarding sexual issues and obtain communication skills, to feel more confident in counselling their patients, which is a future objective of the ARM-Net consortium.

Acknowledgements Open access funding provided by Medical University of Graz. The members of the working group "Follow-up and sexuality" of the ARM-Net consortium would like to thank all participating pediatric surgeons for their contribution in reliably filling out the questionnaire and thus, disclosing personal opinions and current concepts upon managing sexual issues in the treatment of patients with ARM, which helped us to identify the importance of establishing a training for pediatric surgeons towards sexual health and distress.

Members of the ARM-Net consortium not mentioned as coauthors by person: Dalia Aminoff, AIMAR, patient organization, Italy; Piero Bagolan and Barbara Iacobelli, Ospedale Bambino Gesù, Rome, Italy; Paul Broens, University Medical Center Groningen, The Netherlands; Stefan Deluggi and Johanna Ludwiczek, Kepler University Hospital GmbH, Linz, Austria; Maria Fanjul, Hospital Gregorio Maranon, Madrid, Spain; Francesco Fascetti-Leon and Piergiorgio Gamba Dept. Pediatric Surgery, University of Padua, Italy; Carlos Giné, Hospital Vall d'Hebron, Barcelona, Spain; Stefano Giuliani, Great Ormond Street Hospital, London, United Kingdom; Jan Goseman and Martin 
Lacher, University Hospital Leipzig, Germany; Sabine Grasshoff-Derr, Buergerhospital and Clementine Childrens Hospital, Frankfurt, Germany; Stefan Holland-Cunz, University Children's Hospital, Basel, Switzerland; Ernesto Leva and Anna Morandi, Dept. Pediatric Surgery, Policlinico Milano, Milan, Italy; Gabriele Lisi, Santo Spirito Civil Hospital, Pescara, Italy; Omid Madadi-Sanjan, Hospital Hannover, Germany; Igor Makedonsky, Children's Hospital Dnepropetrovsk, Ukraine; Carlo Marcelis, Dept. Human Genetics, Radboudumc, Nijmegen, The Netherlands; Paola Midrio, Dept. Pediatric Surgery, Ospedale Ca'Foncello Treviso, Italy; Onur Ozen, Dept. Pediatric Surgery, Gazi University, Faculty of Medicine, Ankara, Turkey; Alessio PiniPrato, Azienda Ospedaliera Nazionale SS A. e B. e Cesare Arrigo, Italy; Carlos Reck-Burneo, Medical University Wien, Austria; Heiko Reutter, University of Bonn, Germany; Stephan Rohleder, Medical University Hospital, Mainz, Germany; Inbal Samuk, Dept. Pediatric and Adolescent Surgery, Schneider Children's Medical Center, Petach Tikva, Israel; Eberhard Schmiedeke, Clinic Bremen Mitte, Bremen, Germany; Pim Sloots, Dept. Pediatric Surgery, Erasmus Medical Centre, Rotterdam, The Netherlands; Herjan van der Steeg, Dept. of Surgery-Pediatric Surgery, Radboudumc Nijmegen, The Netherlands; Pernilla Stenström, Lund University, Skane University Hospital, Lund, Sweden; Holger Till, Dept. of Pediatric and Adolescent Surgery, Medical University of Graz, Austria; Patrick Volk, Dept. Surgery, University Hospital Heidelberg, Heidelberg, Germany; Tomas Wester, Dept. Pediatric Surgery, Karolinska University Hospital, Stockholm, Sweden.

Author contributions EA, CG and IR conceived the idea. EA drafted the questionnaire, which was further developed with the contribution of all other authors. CG implemented the online questionnaire, collected the data and wrote the method. CG and IR performed the analyses and IR wrote the draft of the results. EA wrote a first draft of the introduction and of the discussion. EA, CG and IR wrote the discussion and contributed to shape the final version of the manuscript. All the other authors commented on the manuscript and approved the final version of the manuscript.

\section{Compliance with ethical standards}

Conflict of interest The authors declare that they have no conflict of interest.

Open Access This article is distributed under the terms of the Creative Commons Attribution 4.0 International License (http://creativeco mmons.org/licenses/by/4.0/), which permits unrestricted use, distribution, and reproduction in any medium, provided you give appropriate credit to the original author(s) and the source, provide a link to the Creative Commons license, and indicate if changes were made.

\section{References}

1. Le Vay S, Valents SH (2003) Human sexuality, chapter 12. Sinauer Associates, Sunderland

2. Schneider W (1987) The psychosocial development of children and adolescents with an organically induced anal incontinence. Prax Kinderpsychol Kinderpsychiatr 36(2):56-62

3. Glass C, Soni B (1999) ABC of sexual health: sexual problems of disabled patients. BMJ 318(7182):518-521. https://doi. org/10.1136/bmj.318.7182.518

4. Grano C, Aminoff D, Lucidi F, Arpante A, Violani C (2008) Selfefficacy, postoperative care satisfaction, body image and sexual functioning in ARM patients. Pediatr Surg Int 24:1201-1205. https://doi.org/10.1007/s00383-008-2233-6

5. Schmidt D, Winter S, Jenetzky E, Zwink N, Schmiedeke E (2012) Sexual function in adults with anorectal malformation: psychosocial adaptation. German Network for Congenital Uro-REctal Malfor- mations (CURE-Net). Pediatr Surg Int 28:789-792. https ://doi.org/10.1007/s00383-012-3119-1

6. Danielson J, Karlbom U, Graf W, Wester T (2017) Outcome in adults with anorectal malformations in relation to modern classification-which patients do we need to follow beyond childhood? J Pediatr Surg 52:463-468. https://doi.org/10.1016/j.jpeds urg.2016.10.051

7. van den Hondel D, Sloots CE, Bolt JM, Wijnen RM, de Blaauw I, Jsselstijn IH (2015) Psychosexual well-being after childhood surgery for anorectal malformation or Hirschsprung's disease. J Sex Med 12(7):1616-1625. https://doi.org/10.1111/jsm.12886

8. Witvliet MJ, van Gasteren S, van den Hondel D, Hartman E, van Heurn LWE, van der Steeg AFW (2018) Predicting sexual problems in young adults with an anorectal malformation or Hirschsprung disease. J Pediatr Surg 53:1555-1559. https://doi. org/10.1016/j.jpedsurg.2018.01.010

9. Hamid CH, Holland AJ, Martin HC (2007) Long term outcome of anorectal malformations; the patients perspective. Pediatr Surg Int 23:97-102. https://doi.org/10.1007/s00383-006-1841-2

10. Davies MC, Liao LM, Wilcox DT, Woodhouse CR, Creighton SM (2010) Anorectal malformations: what happens in adulthood? BJU Int 106:398-404. https://doi.org/10.1111/j.1464410X.2009.09031.x

11. Grano C, Aminoff D, Lucidi F, Violani C (2011) Long-term disease-specific quality of life in adult anorectal malformation patients. Pediatr Surg Int 46:691-698. https://doi.org/10.1016/j. jpedsurg.2010.10.016

12. Hartman EE, Oort FJ, Aronson DC, Sprangers MA (2011) Quality of life and disease-specific functioning of patients with anorectal malformations or Hirschsprung's disease: a review. Arch Dis Child 96:398-406. https://doi.org/10.1136/adc.2007.118133

13. Stenström P, Kockum CC, Benér DK, Ivarsson C, Arnbjörnsson E (2014) Adolescents with anorectal malformation: physical outcome, sexual health and quality of life. Int J Adolesc Med Health 26(1):49-59. https://doi.org/10.1515/ijamh-2012-0111

14. Grano C, Bucci S, Aminoff D, Lucidi F, Violani C (2014) Feelings of depression in people with ARM: the role of critical incidents and perceived difficulties in close and sexual relationships. Pediatr Surg Int 30(8):823-828. https://doi.org/10.1007/s0038 3-014-3532-8

15. McInnes RA (2003) Chronic illness and sexuality. Med J Aust 179(5):263-266

16. de Blaauw I, Wijers CH, Schmiedeke E, Holland-Cunz S, Gamba P, Marcelis CL, Reutter H, Aminoff D, Schipper M, Schwarzer N, Grasshoff-Derr S, Midrio P, Jenetzky E, van Rooij IA (2013) First results of a European multi-center registry of patients with anorectal malformations. J Pediatr Surg 48(12):2530-2535. https://doi.org/10.1016/j.jpeds urg.2013.07.022

17. Kirby D (1998) Mathtech questionnaires: sexuality questionnaires for adolescents handbook of sexuality-related measures. Sage Publications, Thousand Oaks, pp 335-346

18. World Health Organization, UNFPA (2010) Measuring sexual health: conceptual and practical considerations and related indicators. WHO reference number: WHO/RHR/10.12

19. Cairo SB, Gasior A, Rollins MD, Rothstein DH, Delivery of Surgical Care Committee of the American Academy of Pediatrics Section on Surgery (2018) Challenges in Transition of Care for Patients With Anorectal Malformations: A Systematic Review and 
Recommendations for Comprehensive Care. Dis Colon Rectum 61(3):390-399. https://doi.org/10.1097/dcr.0000000000001033

20. Levitt MA, Bischoff A, Breech L, Peña A (2009) Rectovestibular fistula-rarely recognized associated gynecologic anomalies. J Pediatr Surg 44(6):1261-1267. https://doi.org/10.1016/j.jpeds urg.2009.02.046

21. Breech L (2010) Gynecologic concerns in patients with anorectal malformations. Semin Pediatr Surg 19(2):139-145. https://doi. org/10.1053/j.sempedsurg.2009.11.019

22. Greenberg JA, Hendren WH (1997) Vaginal delivery after cloacal malformation repair. Obstet Gynecol 90(4 Pt 2):666-667

23. Greenberg JA, Wu JM, Rein MS, Hendren WH (2003) Triplets after cloacal malformation repair. J Pediatr Adolesc Gynecol 16(1):43-44

24. Kyrklund K, Taskinen S, Rintala RJ, Pakarinen MP (2016) Sexual function, fertility and quality of life after modern treatment of anorectal malformations. J Urol 196(6):1741-1746. https://doi. org/10.1016/j.juro.2016.08.079

25. Konuma K, Ikawa H, Kohno M, Okamoto S, Masuyama H, Fukumoto H (2006) Sexual problems in male patients older than 20 years with anorectal malformations. J Pediatr Surg 41(2):306309. https://doi.org/10.1016/j.jpedsurg.2005.11.004

26. Gott M, Galena E, Hinchliff S, Elford H (2004) "Opening a Can of Worms": GP and practice nurse barriers to talking about sexual health in primary care. J Fam Pract 21(5):528-536. https://doi. org/10.1093/fampra/cmh509

27. Rabathaly PA, Chattu VK (2019) An exploratory study to assess primary care physicians' attitudes toward talking about sexualhealth with older patients in Trinidad and Tobago. J Fam Med Prim Care 8(2):626-633. https://doi.org/10.4103/jfmpc.jfmpc _325_18

28. Karimian Z, Azin SA, Javid N, Araban M, Maasoumi R, Aghayan S, Merghati Khoie E (2018) Reaching consensus: a review on sexual health training modules for professional capacity building. Health Promot Perspect 8(1):1-14. https://doi.org/10.15171/ hpp. 2018.01

29. United Nations Educational, Scientific and Cultural Organization (UNESCO) (2018) International technical guidance on sexuality education: an evidence-informed approach. UNESCO ISBN 978-92-3-100259-5

Publisher's Note Springer Nature remains neutral with regard to jurisdictional claims in published maps and institutional affiliations.

\section{Affiliations}

\section{Eva Elisa Amerstorfer ${ }^{1}$. Caterina Grano ${ }^{2}$. Chris Verhaak ${ }^{3}$ - Araceli García-Vasquez - Marc Miserez $^{5}$. Annemone Radleff-Schlimme ${ }^{6}$. Nicole Schwarzer ${ }^{6} \cdot$ Michel Haanen $^{7} \cdot$ Ivo de Blaauw $^{8}$ - Ekkehart Jenetzky ${ }^{9,10}$. Alida van der Steeg ${ }^{11}$ - Iris A. L. M. van Rooij ${ }^{8,12}$ · ARM-Net consortium}

1 Department of Pediatric and Adolescent Surgery, Medical University of Graz, Auenbruggerplatz 34, 8036 Graz, Austria

2 Department of Psychology, Sapienza University of Rome, Rome, Italy

3 Department of Medical Psychology, Radboudumc Nijmegen, Nijmegen, The Netherlands

4 Department of Pediatric Surgery, University Hospital, 12 de Octubre, Madrid, Spain

5 Department of Abdominal Surgery, University Hospital Gasthuisberg, KU Leuven, Belgium

6 German Self-help Organization for Anorectal Malformations SoMA e.V., Munich, Germany

7 VA-Dutch Patient Organization for Anorectal Malformations, Huizen, The Netherlands
8 Department of Surgery-Pediatric Surgery, Amalia Children's Hospital-Radboudumc Nijmegen, Nijmegen, The Netherlands

9 Department of Child and Adolescent Psychiatry, University Medical Center of the Johannes Gutenberg University, Mainz, Germany

10 Institute of Integrative Medicine, Witten/Herdecke University, Herdecke, Germany

11 Department of Pediatric Surgery, Emma Children's Hospital, AMC and VU University Medical Center, Amsterdam, The Netherlands

12 Department for Health Evidence, Radboud Institute for Health Sciences, Radboudumc Nijmegen, Nijmegen, The Netherlands 